\title{
HER-2, EGFR, Cox-2 and Ki67 expression in lymph node metastasis of canine mammary carcinomas: Association with clinical-pathological parameters and overall survival
}

\author{
M.R. Araújo, L.C. Campos, K.A. Damasceno, C.O. Gamba, E. Ferreira, G.D. Cassali * \\ Departamento de Patologia Geral, Instituto de Ciências Biológicas, Universidade Federal de Minas Gerais, Av. Antônio Carlos 6627, Belo Horizonte, Minas Gerais 31270-901, Brazil
}

\section{A R T I C L E I N F O}

\section{Article history}

Received 3 December 2015

Received in revised form 8 March 2016

Accepted 28 March 2016

\section{Keywords:}

Mammary carcinomas

Canine

Lymph node metastasis

Immunohistochemistry

Survival

\begin{abstract}
A B S T R A C T
Studies about canine mammary tumors based on single molecular markers probably cannot accurately account for the heterogeneity of this disease, and the investigation of multiple molecular alterations in primary tumors and their metastases, in conjunction, has assumed great importance for the understanding of mammary tumor progression. In the present study, we selected 54 primary mammary carcinomas with lymph node metastasis (T1,2,3N1M0), 29 primary mammary carcinomas without metastasis (T1,2,3N0M0), and 25 canine lymph nodes metastasis to evaluate the immunohistochemical expression of HER-2, EGFR, Cox-2 and Ki67 and its association with clinical-pathological parameters and overall survival. Our results found a concordance between the expression of HER-2 (K coefficient: 0.250), Cox-2 (K coefficient: 0.571), and Ki67 (K coefficient: 0.397) and a discordance between EGFR expression (K coefficient: - 0.195) in primary mammary carcinomas and paired lymph node metastasis. Furthermore, a high Ki67 index $(>24 \%)$, large tumor size and the presence of angiolymphatic invasion in canine primary mammary carcinoma with lymph node metastasis plus the presence of extracapsular extension in lymph nodes metastasis were also related to worse prognoses and shorter overall survival $(P<0.05)$. In conclusion, our study demonstrates that primary mammary carcinomas with high expression of HER-2, Cox-2 and Ki67 also show high expression of these markers in paired lymph node metastasis. Moreover, the expression of these molecular markers in lymph nodes metastasis did not demonstrate a prognostic relevance.

(c) 2016 Elsevier Ltd. All rights reserved.
\end{abstract}

\section{Introduction}

As described in both canine and human mammary carcinomas, changes in genes encoding growth factors, growth factor receptors and other regulators of cellular proliferation might play a role in tumorigenesis and therapeutic response (Sorlie et al., 2001). Studies based on single molecular markers cannot accurately account for the heterogeneity of mammary carcinomas (Sorlie et al., 2001; Gama et al., 2008). Given the large number of cellular events involved in cell growth, differentiation, proliferation, invasion and metastasis, the investigation of multiple molecular alterations in conjunction has great relevance for the understanding of mammary carcinoma progression (Argyle and Khanna, 2013). Recently, the evaluation of molecular markers has been also applied to study of lymph nodes metastasis from human and canine mammary tumors (Cho et al., 2008; De Matos et al., 2007; Beha et al., 2012). As in women, regional lymph node status has a major impact on survival in dogs with mammary carcinomas (Hellmén et al., 1993; Yamagami et al., 1996; Chang et al., 2005; Araújo et al., 2015).

\footnotetext{
* Corresponding author.

E-mail address: cassalig@icb.ufmg.br (G.D. Cassali).
}

Mammary carcinomas can develop and progress by a stepwise accumulation of genetic changes, exposure to growth factors, or sex hormones. Among these genetic alterations, amplification of the erbB oncogenes, especially the human epidermal growth factor receptor genes erbB-1 (EGFR, HER-1) and erbB-2 (HER-2, c-erb B 2, HER-2/neu), have been shown to play a fundamental role in the progression of breast cancer and other solid tumors (Slamon et al., 1987; Bacus et al., 1994).

HER-2 gene amplification or HER-2 protein overexpression has been identified in approximately $30 \%$ of human breast cancers and it has been shown to be associated with poor prognosis and prediction of therapeutic response (Slamon et al., 1987; Vogel et al., 2002). In dogs, the prognostic relevance of HER-2 protein expression in mammary carcinomas has yet to be determined. Different authors have shown that the HER-2 protein overexpression in canine mammary carcinomas has been associated with worse prognosis; however, other studies have failed to demonstrate a prognostic relevance of HER-2 protein overexpression (De las Mulas et al., 2003; Hsu et al., 2009; Ressel et al., 2013).

EGFR has been expressed between $20 \%$ to $30 \%$ of human breast cancers and some studies have associated EGFR expression with poor clinical outcome (Aziz et al., 2002; Tsutsui et al., 2002). Elevated 
Table 1

Details of immunohistochemical reagents and methods used in the study.

\begin{tabular}{|c|c|c|c|c|c|c|}
\hline Target antigen & Clone & Manufacturer & Dilution & AR method & Retrieval solution $(\mathrm{pH})$ & Incubation time $(\mathrm{h})$ \\
\hline HER-2 & Polyclonal & Dako & $1: 200$ & Water bath $\left(98^{\circ} \mathrm{C}\right)$ & Citrate (6.0) & 16 \\
\hline EGFR & $31 \mathrm{G} 7$ & Zymed & $1: 50$ & Enzymatic & Pepsin & 16 \\
\hline Cox-2 & SP21 & Labvision & $1: 80$ & Water bath $\left(98^{\circ} \mathrm{C}\right)$ & Citrate (6.0) & 16 \\
\hline Ki67 & Mib-1 & Dako & $1: 25$ & Pressurised heating $\left(125^{\circ} \mathrm{C}\right)$ & Citrate (6.0) & 1 \\
\hline Cytokeratin & AE1AE3 & Dako & $1: 100$ & Water bath $\left(98^{\circ} \mathrm{C}\right)$ & Citrate (6.0) & 1 \\
\hline
\end{tabular}

HER-2: human epithelial grown factor receptor 2; EGFR: epithelial grown factor receptor; Cox-2: cyclooxygenase 2; AR: antigen retrieval.

EGFR serum levels and high expression have also been detected in canine mammary carcinomas using different methodologies, and its expression immunohistochemical has been associated with malignancy (Gama et al., 2009; Bertagnolli et al., 2011; Carvalho et al., 2013; Kim et al., 2013). However, the relevance of EGFR expression as an independent prognostic factor in human or dogs is still controversial (Rampaul et al., 2004; Kim et al., 2013).

Unlike HER-2 and EGFR, other molecules such as Cox-2 and Ki67 are well-recognized prognostic factors in most studies concerning canine mammary carcinomas. Cox-2 expression and a high cell proliferation index (Ki67) have been associated with disease progression, poor prognosis and a shorter survival time in dogs with mammary carcinomas (Dutra et al., 2008; Lavalle et al., 2009; Peña et al., 1998; Millanta et al., 2006; Queiroga et al., 2010; Santos et al., 2013). Nevertheless, data regarding the expression of these biomarkers in lymph node metastasis of canine mammary carcinomas are very limited (Pereira et al., 2009).

Due to the high incidence, clinical importance and new therapies targeted to canine mammary carcinomas, there is an increasing interest in prognostic and predictive biomarkers for the evaluation of tumor progression. In the present study, we evaluated the immunohistochemical expression of HER-2, EGFR, Cox-2 and Ki67 and its association with clinical-pathological parameters and overall survival in canine primary mammary carcinomas with and without metastasis and in paired lymph node metastasis.

\section{Materials and methods}

All procedures were performed under the guidelines and with the approval of the Ethics Committee in Animal Experimentation at the Federal University of Minas Gerais (UFMG), protocol 174/2011.

\subsection{Samples}

Canine mammary carcinomas were selected from the Veterinary School of the Federal University of Minas Gerais and the Laboratory of Comparative Pathology at the Institute of Biological Sciences at the Federal University of Minas Gerais. The cases were staged according to the tumor-node-metastasis (TNM) clinical staging system for canine mammary tumors: this system evaluates tumor size $(\mathrm{T} 1,0-3 \mathrm{~cm}$; $\mathrm{T} 2$, 3-5 cm; T3, >5 cm); the involvement of regional lymph nodes (N0, no metastasis; N1, metastasis), and the presence of distant metastasis (M0, no metastasis; M1, metastasis). Cases were then categorized into five stages: I (T1NOM0); II (T2NOM0); III (T3NOM0); IV (T1,2,3N1M0); and $\mathrm{V}(\mathrm{T} 1,2,3 \mathrm{~N} 0,1 \mathrm{M} 1)$ (Owen, 1980). The presence of lymph node metastasis was diagnosed through hematoxylin-eosin (H\&E) routine staining and was confirmed by immunohistochemistry (IHC) (cytokeratin AE1/AE3 and/or vimentin). To rule out distant metastasis at time of diagnosis, all dogs were underwent thoracic radiography and abdominal ultrasound examination only when serum biochemical changes or increased abdominal size were observed. The cases that displayed systemic metastases were excluded, and 54 cases of primary mammary carcinomas with lymph node metastasis (N1) and 29 primary mammary carcinomas without lymph node metastasis (N0) were selected. Of the 54 cases of primary mammary carcinomas with lymph node metastasis, a total of 99 lymph nodes were evaluated and diagnosed metastasis in 76 lymph nodes. However, in only 25 lymph nodes metastasis (of 25 cases) was possible an evaluation of the tumor markers.

\subsection{Histopathology}

All tumors and lymph node specimens were previously fixed in 10\% neutral buffered formalin, paraffin-embedded and routinely prepared and stained with H\&E. Tumors were classified according to the veterinary histological classification (Cassali et al., 2014; Misdorp et al., 1999). Furthermore, tumors were graded according to the Nottingham Grading System (Elston and Ellis, 1998) and the presence of angiolymphatic invasion (presence of neoplastic cells within the intratumoral and/or peritumoral angiolymphatic vessel spaces) was also evaluated. In the lymph node histological analysis, the presence and the number of lymph node affected with extracapsular extension (ECE) (the presence of neoplastic cells through the capsule of the lymph node metastasis into the perinodal tissue) were evaluated.

\subsection{Immunohistochemistry}

Consecutive $3 \mu \mathrm{m}$ thick sections of primary mammary carcinomas (N0 and N1) and lymph nodes metastasis were cut for IHC analysis, and the antigen was immunodetected using the Dako Advance (HRP) Visualization Method (Dako, Carpinteria, CA, USA). Endogenous peroxidase activity was blocked with a $3 \%$ hydrogen peroxide solution in methyl alcohol. The reagents were applied manually and immunoreactivity was visualized with the chromogen diaminobenzidine (DAB Substrate System, Dako, Carpinteria, CA, USA). Details of the antibodies, dilutions, antigen retrieval procedures, and incubation times used are provided in Table 1. The cytokeratin (CK) AE1AE3 was used to confirm the presence of metastasis. Sections from the HER-2, EGFR and Cox-2, positive canine mammary carcinoma were used as positive controls. Negative controls were assessed using normal serum as the primary antibody.

HER-2 expression were determined by a scoring system established by the American Society of Clinical Oncology, College of American Pathologists (ASCO/CAP) (Wolff et al., 2013) $(0=$ no membrane staining or incomplete and faint/barely perceptible membrane staining in $\leq 10 \%$ of tumor cells; $1+=$ incomplete and faint/barely perceptible membrane staining in $\geq 10 \%$ of tumor cells; $2+=$ incomplete and/or weak/moderate membrane staining in $>10 \%$ of tumor cells or complete and intense membrane staining in $\leq 10 \%$ of tumor cells; and $3+=$ complete and intense membrane staining in $>10 \%$ of tumor cells). In our study, specimens with scores of $0,1+$ and $2+$ were regarded as negative, and score of +3 were defined as positive.

EGFR expression was evaluated by adapted criteria HER-2 assessment (Wolff et al., 2013) and specimens with scores of 0,1+ and $2+$ were regarded as negative, and score of $3+$ were defined as positive.

Positivity for Cox- 2 was indicated by cytoplasmic staining. The number of Cox-2 positive cells was evaluated semi-quantitatively, 
with the distribution score defined by the estimated percentage of positive cells in 5 fields at $400 \times$ magnification: $0=$ absent, $1 \leq 10 \%$ of cells were stained, $2=$ between $10 \%$ and $30 \%$ of cells were stained, $3=$ between $31 \%$ and $60 \%$ of cells were stained, $4=>61 \%$ of cells were stained. To evaluate staining intensity, values from 0 to 3 were

Table 2

Clinical-pathological parameters of the primary mammary carcinomas with lymph node metastasis and without metastasis.

\begin{tabular}{|c|c|c|c|}
\hline & $\mathrm{n}(\%)$ & $\begin{array}{l}\text { Primary mammary } \\
\text { carcinomas with } \\
\text { lymph node } \\
\text { metastasis }^{\mathrm{a}}\end{array}$ & $\begin{array}{l}\text { Primary mammary } \\
\text { carcinomas without } \\
\text { metastasis }^{\text {b }}\end{array}$ \\
\hline $\begin{array}{l}\text { Age }(\text { mean } \pm S D) \\
\text { Breed }\end{array}$ & 79 & $10.88 \pm 2.7$ & $10.51 \pm 2.9$ \\
\hline Crossbreed & 21 & $17(81)$ & $4(19)$ \\
\hline Pure breeds ${ }^{\mathrm{c}}$ & 58 & $34(58.6)$ & $24(41.4)$ \\
\hline \multicolumn{4}{|l|}{ Reproductive status* } \\
\hline Intact & 47 & $27(57.4)$ & $20(42.6)$ \\
\hline Spayed & 21 & $19(90.5)$ & $2(9.5)$ \\
\hline \multicolumn{4}{|l|}{ Tumor localization ${ }^{*}$} \\
\hline Thoracic & 3 & $3(100)$ & $0(0)$ \\
\hline Cranial abdominal & 12 & $11(91.7)$ & $1(8.3)$ \\
\hline Caudal abdominal & 25 & $12(48)$ & $13(52)$ \\
\hline Inguinal & 28 & $16(57.1)$ & $12(42.9)$ \\
\hline Multicentric & 12 & $10(83.3)$ & $2(16.7)$ \\
\hline \multicolumn{4}{|l|}{ Histological type* } \\
\hline Mixed tumor carcinoma & 32 & $11(34.4)$ & $21(65.6)$ \\
\hline Tubular carcinoma & 3 & $3(100)$ & $0(0)$ \\
\hline Papillar carcinoma & 11 & $8(72.7)$ & $3(27.3)$ \\
\hline Solid carcinoma & 17 & $13(76.5)$ & $4(23.5)$ \\
\hline Micropapillary carcinoma & 12 & $12(100)$ & $0(0)$ \\
\hline Special types of carcinoma ${ }^{\mathrm{d}}$ & 2 & $2(100)$ & $0(0)$ \\
\hline Carcinosarcoma & 6 & $5(83.3)$ & $1(16.7)$ \\
\hline \multicolumn{4}{|l|}{ Histological grade ${ }^{* e}$} \\
\hline I & 19 & $7(36.8)$ & $12(63.2)$ \\
\hline II & 44 & $29(65.9)$ & $15(34.1)$ \\
\hline III & 19 & $17(89.5)$ & $2(10.5)$ \\
\hline \multicolumn{4}{|l|}{ Tumor size } \\
\hline $\mathrm{T} 1(<3 \mathrm{~cm})$ & 25 & $12(48)$ & $13(52)$ \\
\hline $\mathrm{T} 2(>3$ and $<5 \mathrm{~cm})$ & 23 & $14(60.9)$ & $9(39.1)$ \\
\hline $\mathrm{T} 3(>5 \mathrm{~cm})$ & 31 & $24(77.4)$ & $7(22.6)$ \\
\hline \multicolumn{4}{|l|}{ Stage $(T N M)^{*}, \mathrm{f}$} \\
\hline I & 13 & $0(0)$ & $13(100)$ \\
\hline II & 9 & $0(0)$ & $9(100)$ \\
\hline III & 7 & $0(0)$ & $7(100)$ \\
\hline IV & 54 & $54(100)$ & $0(0)$ \\
\hline \multicolumn{4}{|l|}{ Angiolymphatic invasion* } \\
\hline Present & 27 & $22(81.5)$ & $5(18.5)$ \\
\hline Absent & 55 & $31(56.4)$ & $24(43.6)$ \\
\hline \multicolumn{4}{|l|}{ Extracapsular extension (ECE) } \\
\hline Present & 22 & $22(100)$ & NA \\
\hline Absent & 32 & $32(100)$ & \\
\hline \multicolumn{4}{|c|}{ Number of lymph nodes with ECE } \\
\hline 1 & 15 & $15(100)$ & NA \\
\hline$\geq 2$ & 7 & $7(100)$ & \\
\hline
\end{tabular}

ECE: extracapsular extension; NA: not applied.

a Some data were not available or an evaluation was not possible for all 54 primary mammary carcinomas with lymph node metastasis.

b Some data were not available for all 29 primary mammary carcinomas without metastasis.

c Pure breeds included Cocker Spaniel, Dachshund, Pitbull, German Shepherd, Pinscher, Schnauzer, Basset Hound, Bichon Frise, Brasilian Fila, Golden Retriever, Siberian Husky and Yorkshire.

d Special types of carcinoma included rich in lipids carcinoma and pleomorphic lobular carcinoma.

e Histological grading based on that of Elston and Ellis (1998).

${ }^{f}$ I (T1NOM0), II (T2NOM0), III (T3NOM0) and IV (T1,2,3N1M0).

$* p<0.05$ significant difference in Exact of Fisher or Chi-square test. attributed: $0=$ absence $(-), 1=$ weak staining $(+), 2=$ moderate staining $(++)$, and $3=$ strong staining $(+++)$. The distribution score and intensity were multiplied to obtain a total score, which ranged from 0 to 12, with scores from 0 to 5 considered low and scores from 6 to 12 considered high (Lavalle et al., 2009).

The cell proliferative index was calculated by counting the number of nuclei positive for Ki-67 staining in a total of 1000 neoplastic cells from each lesion in hot spot areas (Dutra et al., 2008). A cut-off of $24 \%$ positive neoplastic cells for the analysis of Ki67 was used (Peña et al., 1998), with $>24 \%$ Ki67 index considered to be a high cell proliferation index and $<24 \%$ Ki67 index considered to be a low cell proliferation index.

\subsection{Overall survival}

The overall survival was defined as the period (in days) between surgical tumor removal and death due to the tumor. The follow-up period was 1370 days. Animals that died from unknown causes or from causes unrelated to the mammary tumor were censored.

\subsection{Statistical analysis}

Statistical analysis was performed with the software GraphPad Prism 6.0. The D'Agostino \& Pearson omnibus normality test was used to determine if the data were normally distributed. The MannWhitney and unpaired t tests were used to compare the Ki67 index means and size tumor. Possible correlations were investigated by Spearman tests and the associations were described by Chi-square and Fisher's exact tests. The survival curves were calculated with the Kaplan-Meier estimate using the log-rank test. Values were considered statistically significant when $P<0.05$.

The agreement between the HER-2, EGFR, Cox-2 and Ki67 status of primary tumors and paired lymph node metastasis was expressed by the Cohen Kappa coefficient using the software GraphPad Prism QuickCalcs. The relation between the values and the levels of agreement

Table 3

Immunohistochemical data of primary mammary carcinomas without metastasis, primary mammary carcinomas with lymph node metastasis and lymph node metastasis.

\begin{tabular}{lclc}
\hline & $\begin{array}{l}\text { Primary mammary } \\
\text { carcinomas without } \\
\text { metastasis } \\
\text { n/total }(\%)^{\mathrm{a}}\end{array}$ & $\begin{array}{l}\text { Primary mammary } \\
\text { carcinomas with } \\
\text { lymph node metastasis } \\
\text { n/total }(\%)^{\mathrm{b}}\end{array}$ & $\begin{array}{c}\text { Lymph nodes } \\
\text { metastasis } \\
\mathrm{n} / \text { total }(\%)^{\mathrm{c}}\end{array}$ \\
\hline $\begin{array}{l}\text { HER-2 } \\
\text { Negative }\end{array}$ & $23 / 25(88)$ & $44 / 48(91.7)$ & $15 / 23(65.2)$ \\
Positive & $3 / 25(12)$ & $4 / 48(8.3)^{*}$ & $8 / 23(34.8)^{*}$ \\
EGFR & & & \\
Negative & $22 / 26(84.6)$ & $42 / 48(87.5)$ & $22 / 25(88)$ \\
Positive & $4 / 26(15.4)$ & $6 / 48(12.5)$ & $3 / 25(12)$ \\
Cox-2 & & & $19 / 24(79.2)$ \\
Low score & $21 / 23(91.3)$ & $43 / 50(86)$ & $5 / 24(20.8)$ \\
High score & $2 / 23(8.7)$ & $7 / 50(14)$ & $9 / 19(47.4)$ \\
Ki67 index & & & $10 / 19(52.6)$ \\
$<24 \%$ & $15 / 21(71.4)$ & $24 / 48(50)$ & \\
$>24 \%$ & $6 / 21(28.6)$ & $24 / 48(50)$ &
\end{tabular}

a Evaluation of HER-2, EGFR, Cox-2 and Ki67 staining was not possible in all 29 primary mammary carcinomas without metastasis.

b Evaluation of HER-2, EGFR, Cox-2 and Ki67 staining was not possible in all 54 primary mammary carcinomas with lymph node metastasis.

c Evaluation of HER-2, EGFR, Cox-2 and Ki67 staining was not possible in all 25 lymph node metastasis.

d Negative: scores $0,1+$ and $2+$ /Positive: score $3+$.

e Negative: scores $0,1+$ and $2+$ /Positive: score $3+$.

${ }^{f}$ Low score: score 0 to 5/High score: score 6 to 12 .

* Significant difference $(p=0.01)$ in Exact of Fisher test between primary mammary carcinomas and lymph node metastasis. 
were those used by Landis and Koch (1977) with values of 0.00-0.20 indicating slight agreement, values of $0.21-0.40$ indicating fair agreement, values of 0.41-0.60 indicating moderate agreement, values of $0.61-0.80$ indicating substantial agreement, and values of $0.81-1.00$ indicating almost perfect agreement.

\section{Results}

3.1. Clinical-pathological parameters in canine primary mammary carcinomas and regional lymph nodes

The clinical-pathological features of the animals with primary mammary carcinoma without metastasis (NO) and with lymph node metastasis (N1) are summarized in Table 2. The ages of the animals at the time of surgery ranged from 4 to 16 years with a mean of $10.88 \pm$ 2.7 years and $10.51 \pm 2.9$ years in dogs with lymph node metastasis and without metastasis, respectively $(P>0.05)$.

The anatomic location of the carcinomas was more frequent in inguinal glands in dogs with lymph node metastasis $(16 / 28,57.1 \%)$ and in caudal abdominal glands in dogs without metastasis $(13 / 25,52 \%)$ $(P=0.02)$. The thoracic location was observed only in three primary mammary carcinomas with lymph node metastasis.

Higher frequency of carcinoma in mixed tumors $(21 / 32,65.6 \%)$ in dogs without metastasis and of non-mixed tumors and carcinosarcomas $(43 / 51,84.3 \%)$ in dogs with lymph node metastasis $(P<0.001)$ were observed. Grade I (12/19, 63.2\%) was more predominant in primary mammary carcinomas without metastasis and grade II (29/44,65.9\%) and grade III $(17 / 19,89.5 \%)$ were more predominant in primary mammary carcinomas with lymph node metastasis $(P<0.01)$.

The tumor size ranged from 0.5 to $10 \mathrm{~cm}$ (mean of $3.62 \pm 2.49 \mathrm{~cm}$ ) in primary mammary carcinoma without metastasis and from 0.4 to $15 \mathrm{~cm}$ (mean of $5.39 \pm 3.53 \mathrm{~cm}$ ) in primary mammary carcinoma with lymph node metastasis $(P=0.02)$. Angiolymphatic invasion $(22 /$ $27,81.5 \%$ ) was more frequent in primary mammary carcinoma with lymph node metastasis than primary mammary carcinoma without metastasis $(P<0.01)$.

In the lymph node histological analysis, ECE was identified in $42.1 \%$ (32/76) of the lymph nodes metastasis. Additionally, ECE was observed in $40.74 \%(22 / 54)$ of cases of primary mammary carcinomas studied. In $68.2 \%(15 / 22)$ and in $31.8 \%$ (7/22) of these cases, one lymph node and $\geq 2$ lymph nodes with ECE, respectively, were observed.

In primary mammary carcinoma without metastasis, high histological grade correlated with larger tumors $(r=0.45, P=0.01)$ and with the presence of angiolymphatic invasion $(r=0.41, P=0.02)$. Furthermore, the presence of angiolymphatic invasion was correlated with larger tumors $(r=0.47, P=0.01)$.

In primary mammary carcinoma with lymph node metastasis, the presence of angiolymphatic invasion was correlated with high histological grade $(r=0.40, P=0.003)$, with the presence of ECE $(r=0.53$, $p<0.001)$ and with a high number of lymph nodes with ECE $(r=$ $0.48, P<0.001)$.

\subsection{Molecular parameters in primary mammary carcinomas with lymph} node metastasis and without metastasis

The immunohistochemical data of the primary mammary carcinomas with lymph node metastasis and without metastasis are summarized in Table 3. There was no significant difference in the frequency of the expression of all markers between primary mammary carcinomas with lymph node metastasis and without metastasis. The mean expression of the Ki67 index in primary mammary carcinomas with lymph node metastasis and without metastasis was of $26.33 \pm 19.80 \%$ and $19.27 \pm 22.03 \%$, respectively $(P>0.05)$.

In primary mammary carcinomas with lymph node metastasis, the HER-2-positive tumors were positively correlated with a high cell proliferation index $(r=0.32 ; P=0.03)$.
3.3. Relationship between the molecular parameters in primary mammary carcinomas and in lymph node metastasis

The lymph node metastasis immunohistochemical data are also summarized in Table 3. A higher frequency of positivity of HER-2 in lymph node metastasis was observed in relation to primary mammary carcinomas $(P=0.01)$.

The mean expression of Ki67 index in primary mammary carcinomas and in lymph node metastasis was of $26.33 \pm 19.80 \%$ and $28.91 \pm 20.79 \%$, respectively $(P>0.05)$.

The HER-2, EGFR, Cox-2 and Ki67 status of the primary mammary carcinomas and paired lymph nodes metastasis are shown in Table 4. The HER-2 status of the primary mammary carcinomas and paired lymph nodes metastasis was concordant in 15 of 21 cases (71.4\%), including 2 (9.5\%) with positivity to HER-2 and 13 (61.9\%) with negativity to this marker. The Cohen $\mathrm{K}$ coefficient was 0.25 , indicating fair agreement. The EGFR status agreed in 14 of 21 cases (66.7\%), all with negativity to EGFR. However, the Cohen K coefficient was -0.195 , indicating discordance. The Cox-2 status agreed in 17 of 20 cases (85\%), including 3 (15\%) with high score and 14 (75\%) with low score. The Cohen $\mathrm{K}$ coefficient was 0.571 , indicating moderate agreement. Finally, Ki67 status agreed in 12 of 17 cases (70.6\%), including 8 (47.1\%) with a high cell proliferation index and 4 (23.5\%) with a low cell proliferation index. The Cohen $\mathrm{K}$ coefficient was 0.397 , indicating fair agreement.

Additionally, in the correlation analysis, high Cox-2 score in primary mammary carcinomas correlated with high Cox-2 score in lymph nodes metastasis $(r=0.57, P=0.008)$.

The Figs. 1 to 8 illustrating our immunohistochemical results in primary mammary carcinomas and lymph nodes metastasis.

\subsection{Relationship between clinical-pathological and molecular parameters}

In primary mammary carcinomas without metastasis, high histological grade correlated with a high cell proliferation index $(r=0.60, P=$ $0.004)$ and positivity to HER-2 $(r=0.50, P=0.008)$. Furthermore, positivity to EGFR correlated with the presence of angiolymphatic invasion $(r=0.40, P=0.03)$ and with larger tumors $(r=0.52, P=0.007)$.

In primary mammary carcinomas with lymph node metastasis, a high cell proliferation index correlated with presence of ECE ( $r=$ $0.37, P=0.008)$ and a high number of lymph nodes with ECE ( $r=$ $0.39, P=0.006)$.

\subsection{Overall survival}

Survival data were available for 78 dogs. In total, 33 (42.3\%) dogs died because of mammary carcinoma, 5 of which were euthanised because of the disease. Twenty eight (35.9\%) dogs were alive up between 152 and 1095 days post-surgery and 17 (21.8\%) dogs died from causes unrelated to the mammary tumor between 27 and 1370 days postsurgery.

The mean overall survival of dogs with primary mammary carcinoma with lymph node metastasis and without metastasis was $351.56 \pm 272.17$ days and $552.04+327.79$ days $(P=0.004)$, respectively. The log rank test showed longer overall survival of dogs with primary mammary carcinoma without metastasis (not reached the median survival time) than those with lymph node metastasis (median 395 days) $(P=0.002)$ (Fig. 9).

In dogs with primary mammary carcinoma with lymph node metastasis, shorter survival was correlated with high histological grade $(r=-0.32, P=0.02)$, the presence of angiolymphatic invasion $(r=-0.29, P=0.04)$, the presence of ECE $(r=-0.55, P<0.001)$ and high number of lymph nodes with ECE $(r=-0.56, P<0.001)$. However, no correlation between overall survival and clinical pathological parameters was observed in dogs with primary mammary carcinoma without metastasis. 
Table 4

HER-2, EGFR, Cox-2 and Ki67 index status in primary mammary carcinomas and paired lymph node metastasis.

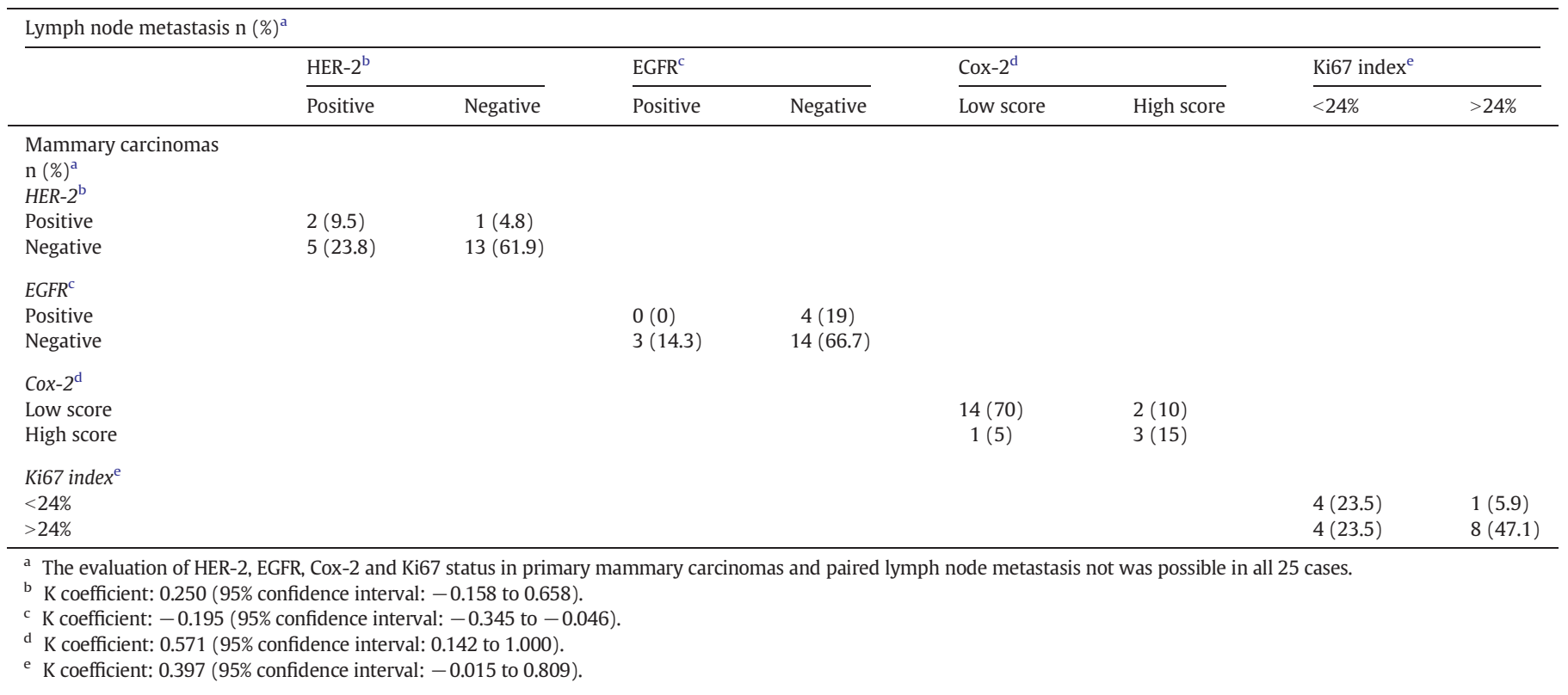

In the survival curve analysis, a statistically significant difference in overall survival was observed with histological grade in dogs with primary mammary carcinoma without metastasis, with tumor size and ECE in dogs with primary mammary carcinoma with lymph node metastasis, and with angiolymphatic invasion in dogs with primary mammary carcinoma with lymph node metastasis and without metastasis.

When evaluated the primary mammary carcinomas with lymph node metastasis, a shorter overall survival was observed in dogs with T2 tumors (median 275 days, $P=0.02$ ) (Fig. 10), with the presence of angiolymphatic invasion (median 188 days, $P=0.01$ ) (Fig. 11) and with the presence of ECE (median 180 days, $P<0.0001$ ) (Fig. 12).

A poor clinical outcome was associated with the presence of angiolymphatic invasion (median 341 days, $P=0.008$ ) (Fig. 13) and with grade III tumors (median 263 days; $P=0.005$ ) in dogs with primary mammary carcinomas without metastasis (Fig. 14).

There were not significant correlations between overall survival and tumor markers expression in dogs with primary mammary carcinomas with lymph node metastasis and without metastasis. These results were confirmed in survival curve analyses $(P>0.05)$. However, a shorter overall survival of animals with high proliferative tumors (median 322 days) in relation to animals with a low proliferative tumors (not reached the median survival time) was observed in primary mammary carcinomas with lymph node metastasis $(P=0.03)$ (Fig. 15). Additionally, when the expression of HER-2, EGFR, Cox-2 and Ki67 was evaluated in lymph node metastasis, no significant difference was observed in the overall survival curve analysis $(P>0.05)$.

\section{Discussion}

Primary mammary carcinomas with metastasis have a significant up-regulation of genes associated with cell cycle regulation, matrix modulation, protein folding and proteasomal degradation, and downregulation of differentiation genes, growth factor pathway genes and regulators of actin organization (Klopfleisch et al., 2010). In our study, the frequency of expression of HER-2, EGFR, Cox-2 and Ki67 did not differ significantly between primary mammary carcinomas with lymph node metastasis and without metastasis.
Some studies have demonstrated that the high frequency of HER-2 protein overexpression in in situ ductal carcinomas of the breast could be an early event in carcinogenesis (Xu et al., 2002). This fact can explain the similar frequency of this marker in the invasive primary carcinomas studied (with lymph node metastasis and without metastasis). However, lymph nodes metastasis (34.8\%) had a significantly higher frequency of positivity to HER-2 compared to primary mammary carcinomas (8.3\%). The epithelial phenotype is an event necessary in the formation of secondary tumors in lymph nodes (Hugo et al., 2007). HER-2 expression occurs mainly in neoplastic epithelial cells in a variety of solid tumors, including mammary tumors (Slamon et al., 1987; Bacus et al., 1994). Furthermore, one study demonstrated a correlation between the phenomenon of epithelial to mesenchymal transition in breast cancer and triple-negative tumors (HER-2 negative, estrogen and progesterone receptors negative) (Jeong et al., 2012). Therefore, our findings may suggest an important role of HER-2 overexpression in the mechanism related to the implantation of neoplastic cells in metastatic niches, such as the mesenchymal to epithelial transition in canine mammary tumors. Moreover, the biologic phenomenon of tumor heterogeneity described in breast cancer could explain the selection of HER-2-positive subclones in the metastasis observed in canine mammary tumors (Malinowsky et al., 2012; Niikura et al., 2012).

Another marker of the same family of human epidermal growth factor receptors, EGFR was not associated with survival in the present study or in the literature (Gama et al., 2009). In our series, we did not find a significant difference in the overall survival of dogs with positivity or negativity for EGFR when evaluated primary mammary carcinomas with lymph node metastasis, primary mammary carcinomas without metastasis and lymph nodes metastasis. Furthermore, the frequency of EGFR expression did not differ between primary mammary carcinomas with lymph node metastasis and without metastasis. EGFR expression has been suggested to be an early event in the carcinogenesis of canine mammary carcinoma, which could explain the findings of the present study (Bertagnolli et al., 2011; Ferreira et al., 2012; Silva et al., 2014). However, an association between the positivity to EGFR and the presence of lymph node metastasis was observed by Guimarães et al. (2014) but not by Gama et al. (2009). These conflicting results may be related to the different analytic methods for evaluating EGFR expression in these studies. In our study, positivity to EGFR was correlated with the 


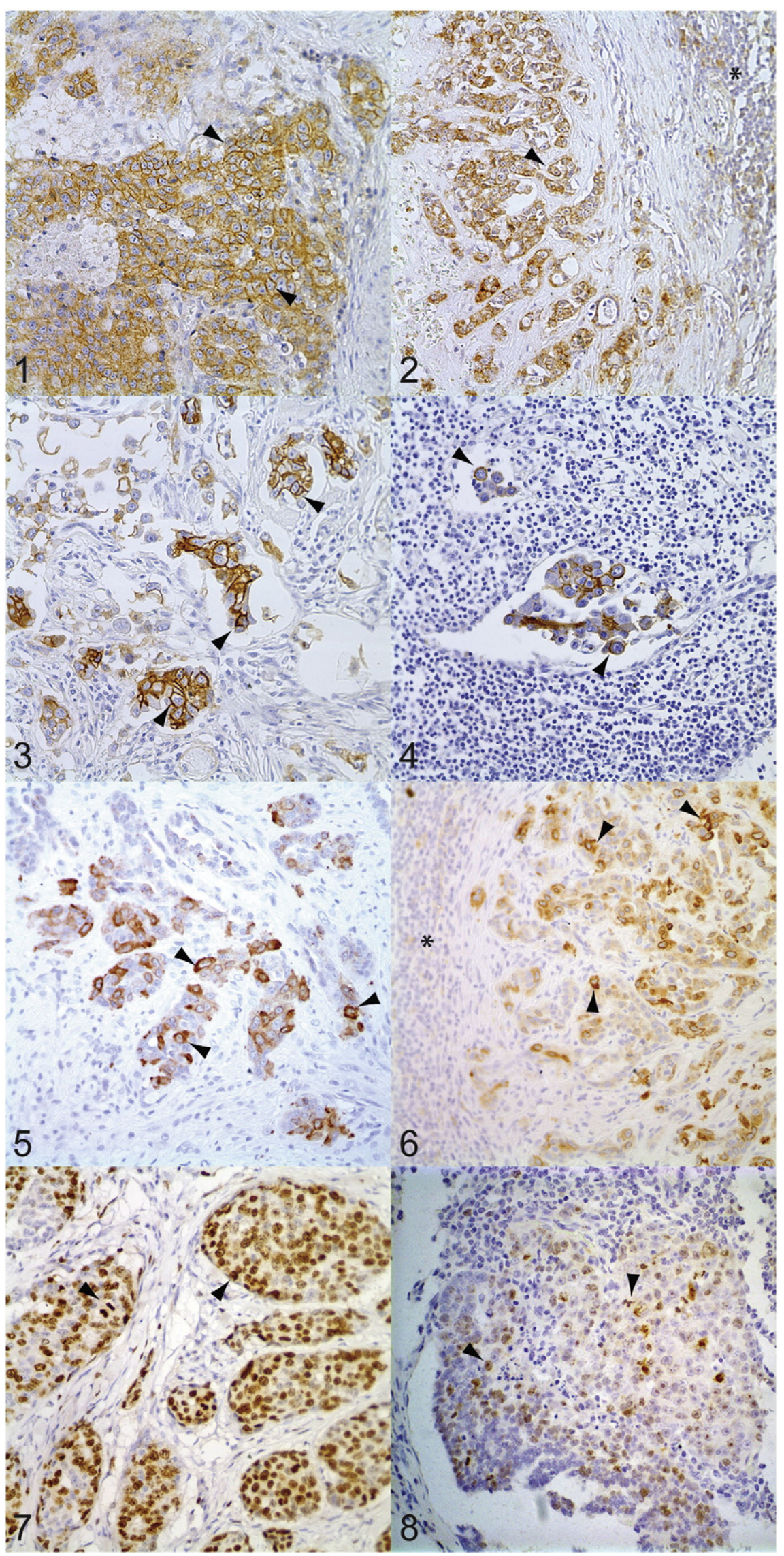


presence of angiolymphatic invasion and larger tumors in primary mammary carcinomas without metastasis. These findings are consistent with previous studies that found a correlation between positivity to EGFR and factors related to biologic aggressiveness such as larger tumors, high grade histologic, the presence of necrosis and the presence of lymphatic invasion (Carvalho et al., 2013; Gama et al., 2009; Kim et al., 2013; Guimarães et al., 2014).

To our knowledge, the present study is the first description of the overall survival of dogs with metastatic carcinoma according to Cox-2 expression in lymph node metastasis. Interestingly, a positive correlation between Cox-2 expression in primary tumors and lymph node metastasis was observed. However, in our study, there was not a significant difference in the frequency of the expression of Cox-2 in primary mammary carcinomas with lymph node metastasis and without metastasis. Few studies have found that the high expression of Cox-2 is associated with the presence of lymph node metastasis in dogs (Guimarães et al., 2014; Queiroga et al., 2010). An analysis of Cox-2 expression in canine metastatic lesions was presented only by Pereira et al. (2009). These authors found that in almost all cases, the labeling intensity of metastatic lesions (pulmonary and lymph node metastasis) was similar or stronger than that of the primary, indicating that Cox-2 metabolic products contributing to metastatic spread of neoplastic cells.

Additionally, the present study did not demonstrate the prognostic relevance of Cox-2 expression when evaluated primary mammary carcinomas with lymph node metastasis, primary mammary carcinomas without metastasis and lymph nodes metastasis. Although the role of Cox-2 and its derived metabolites in tumorigenesis appears to be clear, the prognostic relevance of Cox-2 expression in cancer has not been clearly established (Soslow et al., 2000; Surh et al., 2001; Lavalle et al., 2009). Some studies in veterinary medicine have found that dogs with mammary carcinoma and high Cox-2 expression have a shorter survival time (Millanta et al., 2006; Lavalle et al., 2009; Queiroga et al., 2010). In the current study, only 6 dogs with primary mammary carcinomas with lymph node metastasis had high Cox-2 score, which could explain our findings. Thus, the evaluation of Cox-2 in canine primary tumors and metastases should be investigated in additional studies using different molecular techniques.

In the present study, we demonstrated that Ki67 have prognostic relevance in dogs with primary mammary carcinomas with lymph node metastasis. A shorter overall survival of dogs with a high Ki67 index was also described in previous studies (Peña et al., 1998; Santos et al., 2013). Some multivariable studies, however, reported contrasting results, which may be explained by the fewer number of malignant tumors included in these studies (Löhr et al., 1997; Lee et al., 2004). In our series, a fair agreement between primary mammary carcinomas and paired lymph node metastasis was observed, and most of the concordant cases had high Ki67 index. Interestingly, 23.5\% of the cases had a high cell proliferation index in the primary carcinomas but not in lymph node metastasis and $5.9 \%$ of the cases had a high cell proliferation index in lymph node metastasis but not in primary carcinomas. Using a cut-off of $24 \%$ for Ki67 was not observed significative difference in the frequency between primary mammary carcinomas with lymph node metastasis and without metastasis. Additionally, the frequency also did not differ between the primary carcinomas and lymph node metastasis. Thus, our results may suggest that the primary mammary carcinoma and paired lymph node metastasis are in general made up of cell populations with similar characteristics. Changes in the proliferative activity may occur during growth as a result of the selection of subpopulations with a high metastatic potential, due to the heterogeneity of the primary tumor, genotypic instability, or clonal proliferation during the process of metastasis (Kakeji et al., 1991). Furthermore, the association between the Ki67 index and the presence of lymph node metastasis is controversial in human and canine studies of mammary tumors (De Matos et al., 2006; Leonardi et al., 1992; Peña et al., 1998).

Interesting, in the current study, a significant correlation between a high Ki67 index in primary mammary carcinomas with lymph node metastasis and the presence of ECE and a high number of lymph nodes with ECE was found. Furthermore, dogs presenting lymph nodes with ECE had shorter overall survival in relation to those without ECE. The evaluation of extracapsular extension in the lymph nodes metastasis of dogs with mammary carcinomas is a novel aspect of the present study. This parameter has been studied in human breast cancer and its prognostic relevance has been proven (Yajima et al., 2015). In canine mammary tumors, ECE has not previously been described and our findings suggest that this feature could indicate more extensive disease and may explain the lower survival of these dogs.

\section{Conclusions}

Our study demonstrates that primary mammary carcinomas with lymph node metastasis, presenting a high expression of HER-2, Cox-2 and Ki67 also have a high expression of these markers in paired lymph node metastasis. However, higher frequency of positivity to HER-2 in lymph node metastasis than in primary carcinomas was observed. Furthermore, a high Ki67 index, larger tumor size and the presence of angiolymphatic invasion in canine primary mammary carcinomas with lymph node metastasis and the occurrence of ECE in lymph nodes metastasis were also related to poor prognosis and shorter overall survival. Thus, our results suggest that the molecular parameters studied are associated or correlated with tumor aggressiveness and may be considered in future studies of canine mammary tumors and applied in clinical management.

\section{Conflict of interest statement}

None of the authors have any financial or personal relationships that could inappropriately influence or bias the content of this paper.

\section{Acknowledgements}

This work was financially supported by Conselho Nacional de Desenvolvimento Científico e Tecnológico (CNPq) (470696/2011-7), Coordenação de Aperfeiçoamento de Pessoal de Nível Superior (CAPES), and Fundação de Amparo a Pesquisa de Minas Gerais (FAPEMIG), Brazil.

\section{References}

Araújo, M.R., Campos, L.C., Ferreira, E., Cassali, G.D., 2015. Quantitation of the regional lymph node metastatic burden and prognosis in malignant mammary tumors of dogs. J. Vet. Intern. Med. 29, 1360-1367.

Argyle, D.J., Khanna, C., 2013. Tumor biology and metastasis. In: Withrow, S.J., Macewen's, E.G. (Eds.), Small Clinical Veterinary Oncology, fifth ed. Saunders Elsevier, St Louis, MO, USA, pp. 30-50.

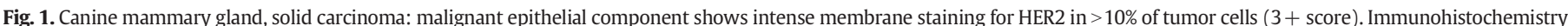

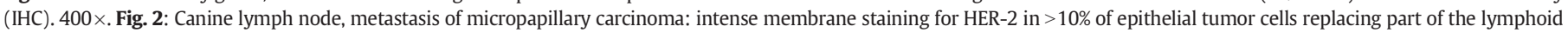

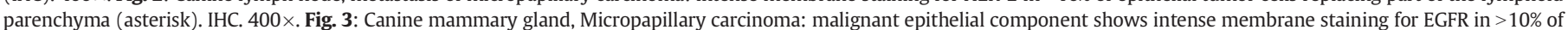

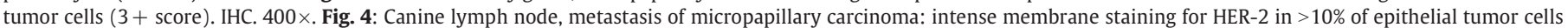

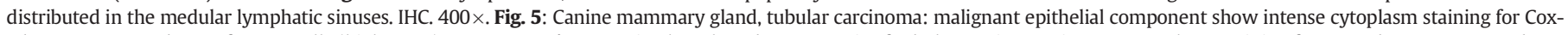

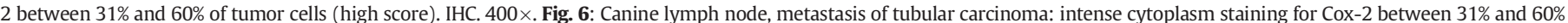

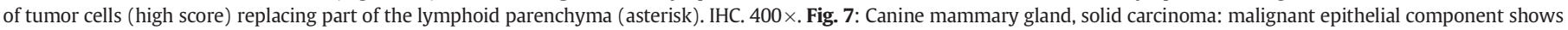

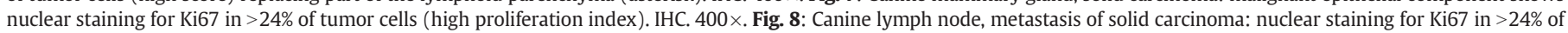
epithelial tumor cells (high proliferation index) distributed in the lymphoid parenchyma. IHC. $400 \times$. 
Primary mammary carcinoma

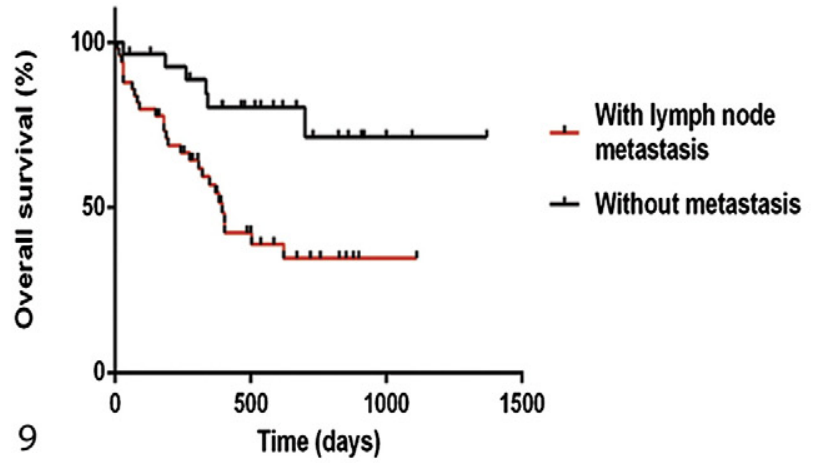

Primary mammary carcinoma with lymph node metastasis

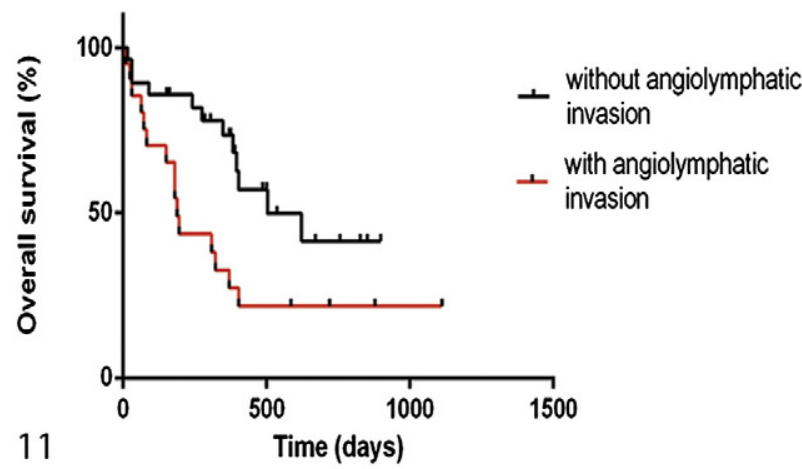

Primary mammary carcinoma without metastasis

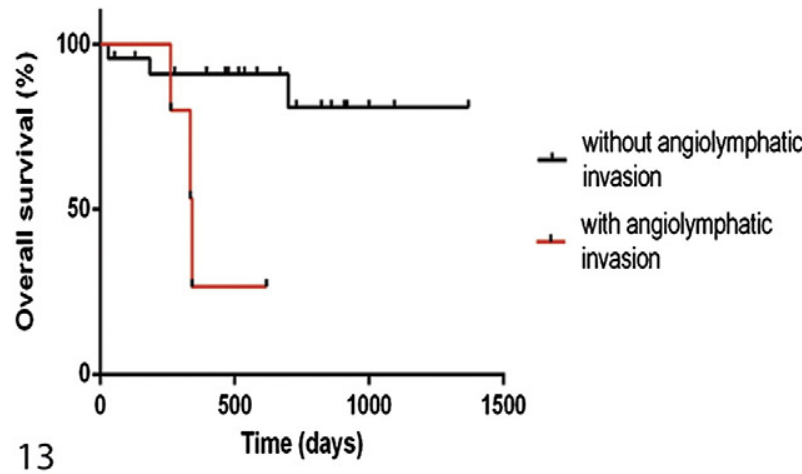

Primary mammary carcinoma with lymph node metastasis

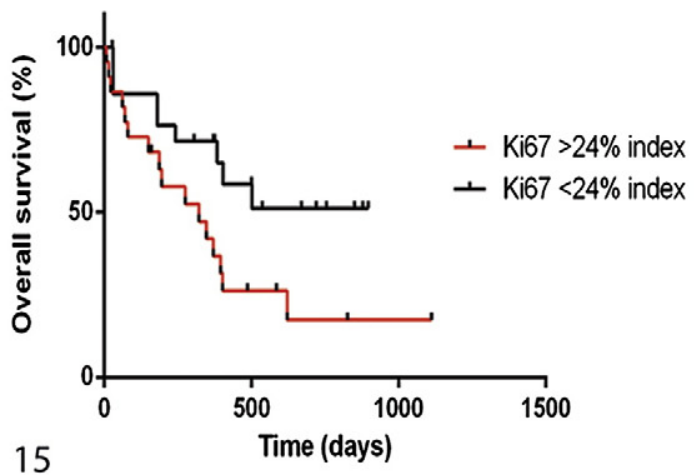

Primary mammary carcinoma with lymph node metastasis

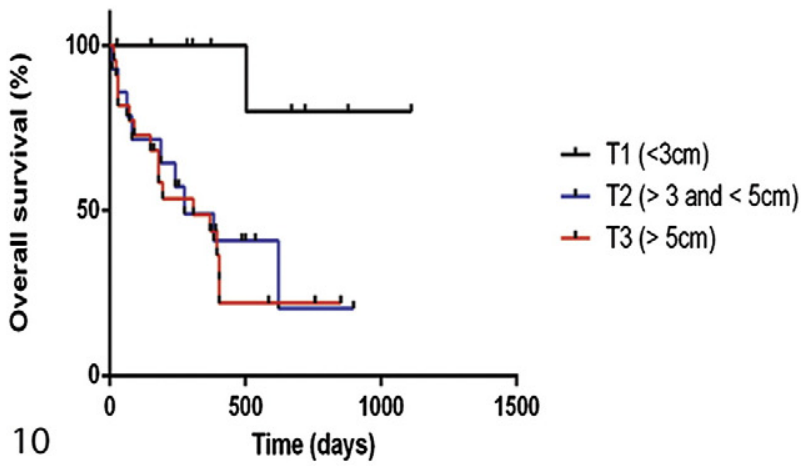

Primary mammary carcinoma with lymph node metastasis

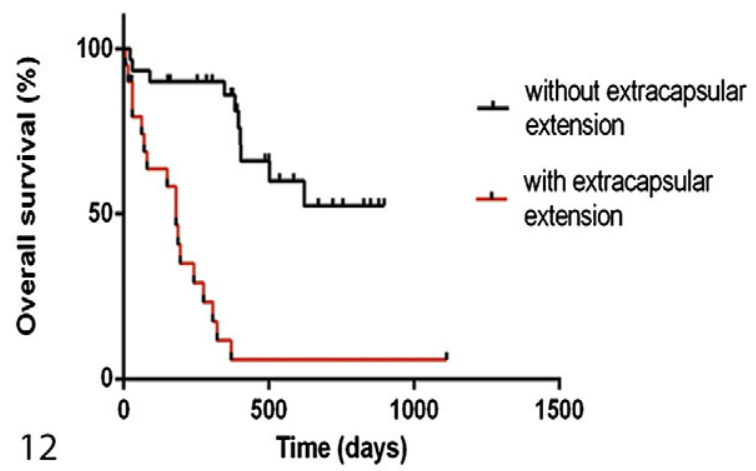

Primary mammary carcinoma without metastasis

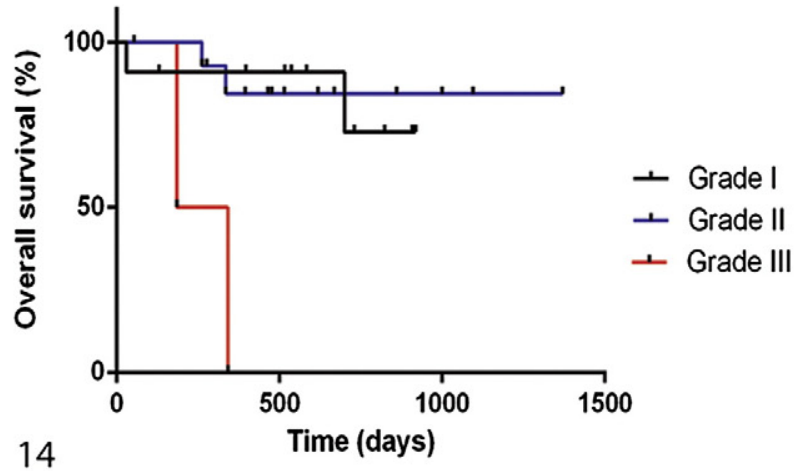


Aziz, S.A., Pervez, S., Kayani, N., Rahbar, M.H., Khan, S., 2002. Epidermal growth factor receptor (EGFR) as a prognostic marker: an immunohistochemical study on 315 consecutive breast carcinoma patients. J. Pak. Med. Assoc. 52, 104-110.

Bacus, S.S., Zelnick, C.R., Plowman, G., Yarden, Y., 1994. Expression of the erbB-2 family of growth factor receptors and their ligands in breast cancers. Implication for tumor biology and clinical behavior. Am. J. Clin. Pathol. 102, S13-S24.

Beha, G., Brunetti, B., Asproni, P., Muscatello, L.V., Millanta, F., Poli, A., Sarli, G., Benazzi, C., 2012. Molecular portrait-based correlation between primary canine mammary tumor and its lymph node metastasis: possible prognostic-predictive models and/ or stronghold for specific treatments? BMC Vet. Res. 8, 219.

Bertagnolli, A.C., Ferreira, E., Dias, E.J., Cassali, G.D., 2011. Canine mammary mixed tumours: immunohistochemical expressions of EGFR and HER-2. Aust. Vet. J. 89, 312-317.

Carvalho, M.I., Guimarães, M.J., Pires, I., Prada, J., Silva-Carvalho, R., Lopes, C., Queiroga, F.L., 2013. EGFR and microvessel density in canine malignant mammary tumours. Res. Vet. Sci. 95, 1094-1099.

Cassali, G.D., Lavalle, G.E., Ferreira, E., Estrela-Lima, A., De Nardi, A.B., Chever, C., Sobral R.A., Amorin, R.L., Oliveira, L.O., Sueiro, F.A.R., et al., 2014. Consensus for the diagnosis, prognosis and treatment of canine mammary tumors - 2013. Braz. J. Vet. Pathol. 7, 38-69.

Chang, S.C., Chang, C.C., Chang, T.J., Wong, M.L., 2005. Prognostic factors associated with survival two years after surgery in dogs with malignant mammary tumors: 79 cases (1998-2002). J. Am. Vet. Med. Assoc. 227, 1625-1629.

Cho, E.Y., Han, J.J., Choi, Y.L., Kim, K.M., Oh, Y.L., 2008. Comparison of Her-2, EGFR and cyclin D1 in primary breast cancer and paired metastatic lymph nodes: an immunohistochemical and chromogenic in situ hybridization study. J. Korean Med. Sci. 23, 1053-1061.

De las Mulas, J.M., Ordás, J., Millán, Y., Fernández-Soria, V., Cajal, S.R., 2003. Oncogene HER-2 in canine mammary gland carcinomas. Breast Cancer Res. Treat 80, 363-367.

De Matos, A.J.F., Lopes, C.C.C., Faustino, A.M.R., Carvalheira, J.G.V., Dos Santos, M.S.A Rutteman, G.R., Gärtner, M.F.R.M., 2006. MIB-1 labelling indices according to clinico-pathological variables in canine mammary tumours: a multivariate study. Anticancer Res. 26, 1821-1826.

De Matos, A.J.F., Lopes, C.C.C., Faustino, A.M.R., Carvalheira, J.G.V., Rutteman, G.R., Gärtner M.F.R.M., 2007. E-cadherin, $\beta$-catenin, invasion and lymph node metastases in canine malignant mammary tumours. Acta Pathol. Microbiol. Immunol. Scand. 115, 327-334.

Dutra, A.P., Azevedo Júnior, G.M., Schmitt, F.C., Cassali, G.D., 2008. Assessment of cell proliferation and prognostic factors in canine mammary gland tumors. Arq. Bras. Med. Vet. Zoot. 60, 1403-1412.

Elston, C.W., Ellis, I.O., 1998. Assessment of histological grade. The Breast, third ed. Churchill Livingstone, London, UK, pp. 365-384.

Ferreira, E., Gobbi, H., Saraiva, B.S., Cassali, G.D., 2012. Histological and immunohistochemical identification of atypical ductal mammary hyperplasia as a preneoplastic marker in dogs. Vet. Pathol. 49, 322-329.

Gama, A., Alves, A., Schmitt, F., 2008. Identification of molecular phenotypes in canine mammary carcinomas with clinical implications: application of the human classification. Virchows Arch. 453, 123-132.

Gama, A., Gärtner, F., Alves, A., Schmitt, F., 2009. Immunohistochemical expression of Epidermal Growth Factor Receptor (EGFR) in canine mammary tissues. Res. Vet. Sci. 87, 432-437.

Guimarães, M.J., Carvalho, M.I., Pires, I., Prada, J., Gil, A.G., Lopes, C., Queiroga, F.L., 2014 Concurrent expression of cyclo-oxygenase-2 and epidermal growth factor receptor in canine malignant mammary tumours. J. Comp. Pathol. 150, 27-34.

Hellmén, E., Bergström, R., Holmberg, L., Spångberg, I.B., Hansson, K., Lindgren, A., 1993. Prognostic factors in canine mammary tumors: a multivariate study of 202 consecutive cases. Vet. Pathol. 30, 20-27.

Hsu, W.L., Huang, H.M., Liao, J.W., Wong, M.L., Chang, S.C., 2009. Increased survival in dogs with malignant mammary tumours overexpressing HER-2 protein and detection of a silent single nucleotide polymorphism in the canine HER-2 gene. Vet. J. 180, 116-123.

Hugo, H., Ackland, M.L., Blick, T., Lawrence, M.G., Clements, J.A., Williams, E.D., Thompson, E.W., 2007. Epithelial-mesenchymal and mesenchymal-epithelial transitions in carcinoma progression. J. Cell. Physiol. 213, 374-383.

Jeong, H., Ryu, Y.J., An, J., Lee, Y., Kim, A., 2012. Epithelial-mesenchymal transition in breast cancer correlates with high histological grade and triple-negative phenotype. Histopathology 60, E87-E95.

Kakeji, Y., Korenaga, D., Tsujitani, S., Haraguchi, M., Maehara, Y., Sugimachi, K., 1991. Predictive value of Ki-67 and argyrophilic nucleolar organizer region staining for lymph node metastasis in gastric cancer. Cancer Res. 51, 3503-3506.

Kim, N.H., Lim, H.Y., Im, K.S., Kim, J.H., Sur, J.H., 2013. Identification of triple-negative and basal-like canine mammary carcinomas using four basal markers. J. Comp. Pathol. $148,298-306$
Klopfleisch, R., Lenze, D., Hummel, M., Gruber, A.D., 2010. Metastatic canine mammary carcinomas can be identified by a gene expression profile that partly overlaps with human breast cancer profiles. BMC Cancer 10, 618 .

Landis, J.R., Koch, G.G., 1977. The measurement of observer agreement for categorical data. Biometrics 33, 159-174.

Lavalle, G.E., Bertagnolli, A.C., Tavares, W.L.F., Cassali, G.D., 2009. COX-2 expression in canine mammary carcinomas correlation with angiogenesis and overall survival. Vet. Pathol. 46, 1275-1280.

Lee, C.H., Lim, J.H., Kim, D.Y., 2004. Mutation and overexpression of p53 as a prognostic factor in canine mammary tumors. J. Vet. Sci. 5, 63-69.

Leonardi, E., Girlando, S., Serio, G., Mauri, F.A., Perrone, G., Scampini, S., Dalla Palma, P., Barbareschi, M., 1992. PCNA and Ki67 expression in breast carcinoma: Correlations with clinical and biological variables. J. Clin. Pathol. 45, 416-419.

Löhr, C.V., Teifke, J.P., Failing, K., Weiss, E., 1997. Characterization of the proliferation state in canine mammary tumors by the standardized AgNOR method with postfixation and immunohistologic detection of Ki-67 and PCNA. Vet. Pathol. 34, 212-221.

Malinowsky, K., Raychaudhuri, M., Buchner, T., Thulke, S., Wolff, C., Höfler, H., Becker, K.F., Avril, S., 2012. Common protein biomarkers assessed by reverse phase protein arrays show considerable intratumoral heterogeneity in breast cancer tissues. PLoS One 7, e40285.

Millanta, F., Citi, S., Della Santa, D., Porciani, M., Poli, A., 2006. COX-2 expression in canine and feline invasive mammary carcinomas: correlation with clinicopathological features and prognostic fmolecular markers. Breast Cancer Res. Treat. 98, 115-120.

Misdorp, W., Else, R.W., Hellmen, E., 1999. Histological Classification of Mammary Tumors of the Dog and the Cat. World Health Organization, Geneva, Switzerland.

Niikura, N., Liu, J., Hayashi, N., Mittendorf, E.A., Gong, Y., Palla, S.L., Tokuda, Y., GonzalezAngulo, A.M., Hortobagyi, G.N., Ueno, N.T., 2012. Loss of human epidermal growth factor receptor 2 (HER2) expression in metastatic sites of HER2-overexpressing primary breast tumors. J. Clin. Oncol. 30, 593-599.

Owen, L.N., 1980. TNM Classification of Tumors in Domestic Animals. first ed. World Health Organization, Geneva, Switzerland, pp. 26-32.

Peña, L.L., Nieto, A.I., Pérez-Alenza, D., Cuesta, P., Castaño, M., 1998. Immunohistochemical detection of Ki-67 and PCNA in canine mammary tumors: relationship to clinical and pathologic variables. J. Vet. Diagn. Investig. 10, 237-246.

Pereira, P.D., Lopes, C.C., Matos, A.J.F., Santos, M., Gärtner, F., Medeiros, R., Lopes, C., 2009. COX-2 expression in canine normal and neoplastic mammary gland. J. Comp. Pathol. $140,247-253$.

Queiroga, F.L., Pires, I., Lobo, L., Lopes, C.S., 2010. The role of Cox-2 expression in the prognosis of dogs with malignant mammary tumours. Res. Vet. Sci. 88, 441-445.

Rampaul, R.S., Pinder, S.E., Wencyk, P.M., Nicholson, R.I., Blamey, R.W., Robertson, J.F., Ellis, I.O., 2004. Epidermal growth factor receptor status in operable invasive breast Cancer is it of any prognostic value? Clin. Cancer Res. 10, 2578.

Ressel, L., Puleio, R., Loria, G.R., Vannozzi, I., Millanta, F., Caracappa, S., Poli, A., 2013. HER-2 expression in canine morphologically normal, hyperplastic and neoplastic mammary tissues and its correlation with the clinical outcome. Res. Vet. Sci. 94, 299-305.

Santos, A.A., Lopes, C.C., Ribeiro, J.R., Martins, L.R., Santos, J.C., Amorin, I.F., Gärtner, F., Matos, A.J., 2013. Identification of prognostic factors in canine mammary malignant tumours: a multivariable survival study. BMC Vet. Res. 9, 1.

Silva, I.L.D., Dias, A.P.M., Bertagnolli, A.C., Cassali, G.D., Ferreira, E., 2014. Analysis of EGFR and HER-2 expressions in ductal carcinomas in situ in canine mammary glands. Arq. Bras. Med. Vet. Zoot. 66, 763-768.

Slamon, D.J., Clark, G.M., Wong, S.G., Levin, W.J., Ullrich, A., McGuire, W.L., 1987. Human breast cancer: correlation of relapse and survival with amplification of the HER-2/ neu oncogene. Science 235, 177-182.

Sorlie, T., Perou, C.M., Tibshirani, R., Aas, T., Geisler, S., Johnsen, H., Hastie, T., Eisen, M.B., Van de Rijn, M., Jeffrey, S.S., et al., 2001. Gene expression patterns of breast carcinomas distinguish tumor subclasses with clinical implications. Proc. Natl. Acad. Sci. U. S. A. $98,10869-10874$.

Soslow, R.A., Dannenberg, A.J., Rush, D., Woerner, B.M., Nasir Khan, K., Masferrer, J., Koki, A.T., 2000. COX-2 is expressed in human pulmonary, colonic, and mammary tumors. Cancer 89, 2637-2645.

Surh, Y.J., Chun, K.S., Cha, H.H., Han, S.S., Keum, Y.S., Park, K.K., Lee, S.S., 2001. Molecular mechanisms underlying chemopreventive activities of anti-inflammatory phytochemicals: down-regulation of COX-2 and iNOS through suppression of NF- $\kappa$ B activation. Mutat. Res. 480-481, 243-268.

Tsutsui, S., Ohno, S., Murakami, S., Hachitanda, Y., Oda, S., 2002. Prognostic value of epidermal growth factor receptor (EGFR) and its relationship to the estrogen receptor status in 1029 patients with breast cancer. Breast Cancer Res. Treat. 71, 67-75.

Vogel, C.L., Cobleigh, M.A., Tripathy, D., Gutheil, J.C., Harris, L.N., Fehrenbacher, L., Slamon, D.J., Murphy, M., Novotny, W.F., Burchmore, M., et al., 2002. Efficacy and safety of trastuzumab as a single agent in first-line treatment of HER2-overexpressing metastatic breast cancer. J. Clin. Oncol. 20, 719-726.

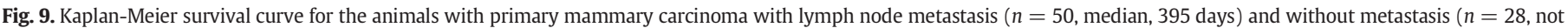

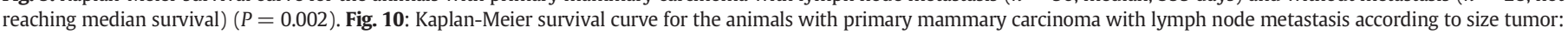

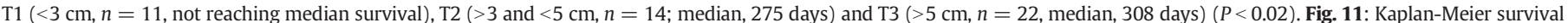

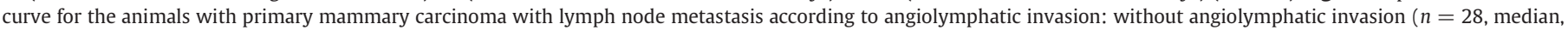

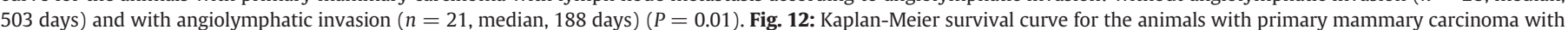

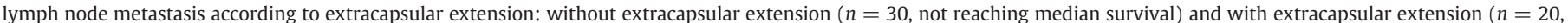

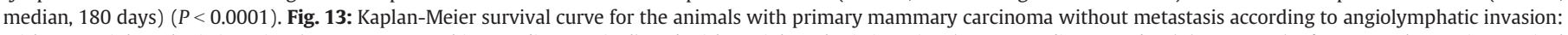

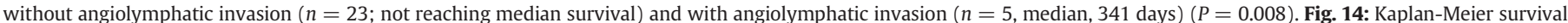

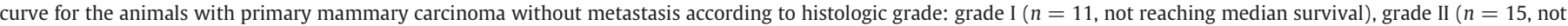

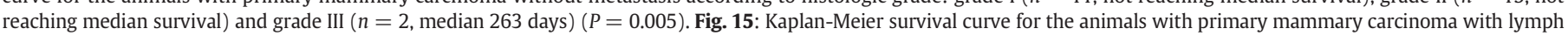
node metastasis according to the Ki67 index: Ki67 $>24 \%$ index $(n=22$, median 322 days $)$ and Ki67 $<24 \%$ index $(n=22$, not reaching median survival $)(P=0.03)$. 
Wolff, A.C., Hammond, M.E.H., Hicks, D.G., Dowsett, M., McShane, L.M., Allison, K.H., Allred, D.C., Bartlett, J.M.S., Bilous, M., Fitzgibbons, P., et al., 2013. Recommendations for human epidermal growth factor receptor 2 testing in breast cancer: American Society of Clinical Oncology/College of American Pathologists clinical practice guideline update. J. Clin. Oncol. 31, 3997-4013.

Xu, R., Perle, M.A., Inghirami, G., Chan, W., Delgado, Y., Feiner, H., 2002. Amplification of Her-2/neu gene in Her-2/neu-overexpressing and-nonexpressing breast carcinomas and their synchronous benign, premalignant, and metastatic lesions detected by FISH in archival material. Mod. Pathol. 15, 116-124.
Yajima, R., Fujii, T., Yanagita, Y., Fujisawa, T., Miyamoto, T., Hirakata, T., Tsutsumi, S. Lijima, M., Kuwano, H., 2015. Prognostic value of extracapsular invasion of axillary lymph nodes combined with peritumoral vascular invasion in patients with breast cancer. Ann. Surg. Oncol. 22, 52-58.

Yamagami, T., Kobayashi, T., Takahashi, K., Sugiyama, M., 1996. Prognosis for canine malignant mammary tumors based on TNM and histologic classification. J. Vet. Med. Sci. $58,1079-1083$. 